\title{
Cuerpo y símbolo en Ernst Cassirer: la función ORGÁNICA EN LA CONFORMACIÓN DEL MUNDO CULTURAL
}

\author{
Gustavo Esparza ${ }^{1}$
}

\begin{abstract}
Resumen: En este trabajo se estudia la función del cuerpo humano y su relación con la cultura en la Filosofía de formas simbólicas de Ernst Cassirer. Según el neokantiano, la relación cuerpo-alma conforma la relación básica para el desarrollo de la "Fenomenología del conocimiento", sin embargo, a través del proceso dialéctico es necesario negar la forma material para lograr la forma espiritual de la cultura por lo que la organicidad corporal, al parecer, debe anularse en esta progresión dialéctica. El objetivo es analizar y describir cómo la forma del cuerpo fundamenta el escenario mítico expresivo, pero también la fase representativa del lenguaje y la significativa de la ciencia, permitiendo la unidad en todo el proceso de la construcción del conocimiento y la forma de la cultura.
\end{abstract}

Palabras Clave: Cuerpo. Símbolo. Conocimiento. Función orgánica. Función cultural. Uexküll. Cassirer.

\section{INTRODUCCIÓN}

El objetivo del presente trabajo es profundizar los conceptos de "cuerpo" y "cultura" en la filosofía de Ernst Cassirer ${ }^{2}$ para elucidar la función que media entre ambos en el proceso de construcción del conocimiento. El interés es mostrar que la "forma" orgánica del cuerpo, dentro de la filosofía del neokantiano, es condición material para el desarrollo de la "forma" espiritual de la cultura.

\footnotetext{
${ }^{1}$ Profesor investigador del Departamento de Humanidades, Universidad Panamericana, Aguacalientes - Mexico. (D) https://orcid.org/0000-0002-9470-6519 E-mail: gaesparza@up.edu.mx

${ }^{2}$ Las obras de Ernst Cassirer se citarán de acuerdo al siguiente criterio: SuF, (Cassirer 1910/1953), PSF, I (Cassirer, 1998a/2010), PSF, II (Cassirer, 1998b/2010), PSF, III (Cassirer, 1998c/2010), PSF, IV, (1996), $Z L K$ (Cassirer, 2005) y $A E M$, (Cassirer, 1945). Las obras del autor se citarán de acuerdo a las ediciones traducidas, por tanto, aparecerán primero los números de la obra consultada y en segundo lugar las páginas de las versiones originales. En las notas a pie de página se conserva el idioma original de la obra.
}

http://doi.org/10.1590/0101-3173.2020.v43esp.16.p205

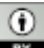

This is an open-access article distributed under the terms of the Creative Commons Attribution License. 
El presente estudio se centra en la Filosofía de las formaciones simbólicas, donde el autor solventa dos proyectos: primero, la construcción de una teoría del conocimiento que se sustenta de las manifestaciones culturales como expresión de la naturaleza humana; segundo, la identificación de los recursos culturales del autoconocimiento como fin general de la Filosofía. En el contexto de ambas tareas, el neokantiano plantea que la forma de la cultura depende de la "naturaleza animal" del ser humano, no por su cualidad de ser vivo sino por las capacidades de éste para "simbolizar".

Para Cassirer, el ser humano se encuentra limitado por la constitución orgánica que le caracteriza como un ser viviente; a pesar de ello, dicha limitación no se entiende como sujeción del organismo al entorno material, sino que se traduce en la capacidad psicológica y relacional de los individuos para vincularse a su círculo funcional. De ese modo, el ser humano, a la vez que opera como un ser orgánico que vive en un mundo, se conoce a sí mismo como un animal simbolizante cuyo espacio vital es la cultura ( $Z L K$, p. 32-49; $A E M$, p. 41-44). El de Breslavia, a lo largo de su obra, muestra que los símbolos son el producto de una coordinación funcional de relaciones que al interactuar y vincularse con una forma general de la serie se traducen progresivamente en manifestaciones culturales (SuF, p. 3-26/3-34).

Antes de presentar el problema es importante presentar dos ideas centrales del autor. Primera en el volumen dedicado a la Fenomenología del conocimiento se sostiene que el "fenómeno originario de la expresión" (das Urphänomen des Ausdrucks) surge como la identidad y el fundamento que une la dualidad cuerpo-alma (PSF, III, p. 124/113-114), por lo que el conocimiento se funda en el marco de esta relación de unidad. Sin embargo, tanto el "yo" del sujeto como el "tú/ello" del objeto, son dualidades independientes que se unificarán en la progresión del proceso de simbolización cultural. Segunda, las funciones simbólicas son presentadas por el autor como una "crítica de la cultura" (PSF, I, p. 20/9) que se construye como parte del proceso de simbolización formal, el cual, se dijo, depende de la unidad del cuerpo-alma.

De acuerdo con esto, entonces, la relación de unidad entre el cuerpo y la cultura, parece admitir el siguiente proceso: la relación cuerpo-alma es el fundamento de las formaciones simbólicas, de tal modo que cada símbolo se entiende como una expresión aquella identidad (cuerpo/alma), lo que, a su vez, permite la conformación del espacio cultural. Como se aprecia, el alma debería aparecer como el recurso de unificación entre el cuerpo y la cultura. Sin embargo, el problema es que para Cassirer la función entre el 
cuerpo y la cultura no admite intermediaciones, sino que se expresa como unidad originaria en todas las fases de la Fenomenología del conocimiento. En lo consecuente se analiza cómo es posible la expresión e interrelación del cuerpoalma-cultura en el proceso general de construcción del conocimiento dentro de la filosofía de este neokantiano.

\section{El PROBLEMA DE LA EXPERIENCIA EN EL NEOKANTISMO}

Antes de ingresar a la revisión puntual del problema en la obra de Cassirer, es importante preguntarse ¿Por qué el cuerpo ocupa un lugar en la reflexión filosófica de este neokantiano? ¿Existe algún contexto teórico en el que esta temática tenga cabida dentro de la tradición kantiana? Estas preguntas son importantes para entender la aportación específica que realiza el filósofo de Breslavia en relación a un tema que había ocupado una buena parte de las investigaciones filosófico biológicas de aquella época.

Como contexto histórico general hay que tener presente el detallado estudio de Klaus Köhnke (2011) en donde describe la evolución de la filosofía kantiana hasta constituirse como filosofía neokantiana. Para este autor, una de las determinaciones fundamentales de este movimiento surge de la interpretación que hace Herman Cohen (2015) de la Crítica de la Razón Pura, la cual, a pesar de presentarse como una interpretación rigorista y exegética de la obra de Kant, se recibe como una oposición a la hasta entonces convención filosófica alcanzada. Cohen, partiendo de la $K r V$, deduce una "crítica de la experiencia" (Könke 2011, p. 282-310), lo que en principio constituye un contrasentido. Si bien Cohen no pretendía reconstruir la interpretación filosófica sobre los postulados kantianos, sitúa como problema central la necesidad de reflexionar sobre las condiciones que hacen posible la "razón pura". La experiencia para Cohen ya no es la mera posibilidad de percibir, sino el conjunto de condiciones que hacen posible la razón pura, por ello ahora se invierte el procedimiento reflexivo para determinar sus condiciones de viabilidad de tal modo que es el papel de la ciencia, a través de sus experimentos, establecer la diferencia entre la percepción del sujeto y la determinación de lo experiencia como recurso base de conocimiento.

Paul Natorp (1975) continuará con estas reflexiones al cuestionar la viabilidad del conocimiento y las condiciones generales que posibilitaban su constitución. Tanto en la famosa conferencia Kant y la Escuela de Marburgo como en su Curso de Pedagogía Social el autor establece algunos lineamientos 
centrales sobre el problema de las condiciones a través de las cuales es posible conocer. En la conferencia del autor, además de aceptar los postulados cohentianos generales, propone reflexionar filosóficamente sobre "hechos patentes, históricamente determinables de la ciencia” (NATORP, 1975, p. 80), para con ello recurrir a hechos objetivos y materiales. Además de esto, el propio autor manifestará que la reflexión filosófica objetiva y sistematiza la reflexión pedagógica de tal modo que considera deber de la pedagogía apoyarse de los principios generales buscados por la filosofía. De ese modo, la reflexión sobre la constitución de la formación humana descansa en la comprensión de la "objetividad" del ser humano. La filosofía de Marburgo había situado como uno de sus diversos problemas la investigación de la naturaleza de todas las cosas a la luz de un "método trascendental" (NATORP, 1975, p. 79-81) cuyo procedimiento convencional es la investigación de la experiencia y operatividad de los recursos a que hacen posible el conocimiento.

Cassirer refinará estas investigaciones de tal modo que la reflexión crítica del cuerpo aparece como uno de las múltiples tareas que debían ser resueltas con el fin de comprender los procesos básicos que garantizaban las condiciones críticas del conocimiento. Esto se advierte desde Concepto Sustantivo y Concepto Funcional $(\mathrm{SuF})$, aunque parte de una investigación sobre el papel que desarrolla la "función matemática" en la constructividad del conocimiento, allí se detallan las condiciones de relacionalidad implicadas en la constitución del vínculo entre la estructura material de los objetos estudiados y una ley general que los dota de sentido. El avance de las ciencias a través de la historia del pensamiento es el recurso de objetividad a través del cual es posible evaluar la forma del mundo natural y el conocimiento que de ello se deriva. De ese modo es comprensible que el cuarto volumen sobre El problema del conocimiento (CASSIRER 1998c, p. 148-262) reflexionara sobre el proceso histórico en el que la Biología había aparecido como una ciencia que tenía por objeto el estudio de la "vida" en oposición al estudio de la "realidad", propio de la Física 3

Este análisis histórico es fundamental para la comprensión del problema que aquí se aborda. Si las ciencias tenían como interés el estudio de la "experiencia" a través de la organización y sistematización de "experimentos" que dieran cuenta de las condiciones críticas del conocimiento objetivo del mundo, entonces, era necesario indagar filosóficamente sobre el marco de

\footnotetext{
${ }_{3}^{3}$ Para profundizar en las implicaciones de esta diferenciación conceptual entre Biología y Física: (Esparza 2018, p. 130-133)
} 
resultados científicos para comprender la forma histórica en la que se había desarrollado El problema del conocimiento. Sin embargo, para el propio autor la forma del conocimiento y sus condiciones de posibilidad seguirían estando vinculadas a su agente, de tal modo que no es sólo posible indagar sus condiciones "objetivas" dejando de lado las condiciones "subjetivas" en las que se desarrollaba este proceso.

Esta necesidad sería considerada por Cassirer en sus trabajos sobre Helmholtz y Uexküll en donde se aprecia que la resolución del problema del conocimiento debía primero investigar las condiciones fisiológicas a través de las cuales el ser humano percibía los fenómenos del mundo. Para ellos, las condiciones críticas de la percepción no dependían exclusivamente de una naturaleza rígidamente dispuesta en el ser humano, sino que debían comprenderse las condiciones biológicas en las cuales este proceso ocurría. Para cumplir tal fin, era necesario que la filosofía se apoyara de la investigación médica como recurso epistemológico para resolver esta cuestión. Estas investigaciones sobre el organismo irán permeando los estudios sobre el lenguaje, el mito y la ciencia a través de la obra magna de Cassirer. Sobre este aspecto, otros estudios al respecto han mostrado que

La antropología propuesta por Ernst Cassirer, y particularmente, la definición de "animal simbólico" encuentra en los estudios biológicos y la patología de la acción y el lenguaje, una base teórica para el desarrollo de una nueva definición del ser humano. Esta propuesta particular del neokantiano corresponde a un resultado de un análisis biológico de la estructura y conducta humana. Aunque se podría pensar que la PSF, en su conjunto, fundamenta la naturaleza simbólica y animal del ser humano, no es posible tal logro ajustándose a una perspectiva puramente epistemológica (ESPARZA 2019, p. 141).

Como se aprecia, la reflexión sobre las condiciones que hacen posible el conocimiento aparecen en el dilema de cuál es la forma de relación que existe entre la estructura orgánica del individuo y el entorno cultural en el que se desarrolla. El objetivo central de esta respuesta es la profundización de los recursos indispensables para solventar el dilema propuesto por Cohen (2015), es decir: ¿Cuáles son las condiciones críticas (naturales, matemáticas, históricas) que hacen posible el conocimiento? En lo que resta del texto se estudiará el problema y solución ofrecidos por Cassirer a la pregunta sobre la experiencia del mundo natural y su relación con el conocimiento puro. Hacia el final se resaltará que si bien el problema de partida indaga cómo 
se vincula el "cuerpo" y el "alma” en la construcción del conocimiento, la perspectiva cassireriana innovará en el tratamiento de la cuestión al introducir a la "cultura" como una condición sine qua non en la construcción de una red epistemológica de relaciones conceptuales.

\section{LA UNIDAD CUERPO-ALMA COMO FUNDAMENTO CULTURAL}

Al considerar como punto problemático de partida la dificultad de unificar el cuerpo, el alma y la cultura podemos identificar los siguientes resultados en el estado de la cuestión. Primero, para Roberto González (2015) la unidad "cuerpo-alma" opera como el momento primario en la construcción simbólica del conocimiento; con esta relación se posibilita la percepción del universal por parte del ser humano singular, de tal modo que, el entrelazamiento apunta a la capacidad básica y progresiva con la cual distinguir entre el cuerpo como el sujeto que conoce y lo ajeno como objeto conocido. González establece que el cuerpo es un factor básico en la construcción simbólica del mundo. Sin embargo, aunque el autor advierte que esta unidad se mantiene a lo largo de la fenomenología, el análisis se centra en su fase expresiva.

Por otra parte, Graciela Ralón (2002) amplia el análisis asumiendo el problema de la percepción. Ella ubica la unidad del cuerpo-alma como fundamento primario de la percepción al sostener que "aparece como un comportamiento subjetivo determinado por una función simbólica específica” (RALÓN, 2002, p. 36), de tal modo que el acto perceptivo se encuentra supeditado a un símbolo. Así, la progresión de la percepción se caracterizará por una pérdida gradual de los fundamentos que inicialmente la sostienen:

Si bien es cierto que [en la fase de representación] nunca se pierde la conexión con la experiencia expresiva primaria... no se puede olvidar que entre ambas existe una infranqueable diferencia funcional ya que no es posible derivar una de otra (RALÓN, 2002, p. 41).

Ralón plantea que esta distancia "infranqueable" ocurre tanto en los modos de percibir, como en los recursos para hacerlo; mientras que en el primer estadio el cuerpo es la condición material a través de la cual se aprehende un fenómeno, en el segundo, la forma (Gestalt) de la percepción se torna la condición espiritual que hace posible el propio acto de percepción. Con esto, la autora sostiene que el cuerpo, para Cassirer, en lugar de operar como unidad 
de síntesis orgánica de las percepciones a lo largo de todo el proceso, es una fase unificante del estadio expresivo.

Este planteamiento puede contrastarse con lo dicho por Krois (2007) y Rosengren (2007). A decir de los autores, la naturaleza del conocimiento adquiere una conciencia progresiva y gradual de sentido espiritual hasta reconocer a los objetos culturales como manifestaciones de un animal simbolizante. De ese modo, la unidad de la cultura puede definirse como "encarnación cognitiva" (emodied cognition) que afirma tanto la estructura corporal como la constitución espiritual de los objetos culturales. Para ambos autores la cultura es una representación funcional de la naturaleza orgánica y espiritual del ser humano.

Christian Möckel (2011) ha especificado esta relación al definirla como forma viviente (Lebendige Formen), la cual se mantiene y subyace en las expresiones del conocimiento. Así, las manifestaciones culturales son formas epistémicas en las que se ratifica la unidad del cuerpo y el alma. Möckel resalta que a través del concepto "Forma de la ciencia" (Formwissenschaft) se reflejan las actividades culturales superiores como expresión del organismo viviente que las impulsa, por ello, expresiones como "organismos científicos" o "cuerpos de trabajo", se aplican como una extensión lingüística de la forma del conocimiento humano. Hay que recordar que el concepto de forma subraya la unidad de vinculación entre las individualidades en relación a un marco general de sentido (PSF, III, p. 331-367/323-361). Así, el concepto de la ciencia como forma delimita un espacio vital de sentido en el que operan los sujetos de la ciencia.

Como se aprecia, existe un avance en la comprensión de la relación cuerpo, alma y cultura, sin embargo, aún no se aclara cómo la estructura orgánica del animal simbólico se mantiene en todas las formas abstractas de la cultura. Para mostrar la armonía entre aquella tríada sostendré que la unidad funcional propuesta por Cassirer a lo largo de su obra, se apoya del concepto de “círculo funcional" propuesto por el biólogo estonio Jakob von Uexküll (1926).

\section{CUERPO Y CULTURA: UNA APROXIMACIÓN ORGÁNICA AL SÍMBOLO}

La teoría del cuerpo desarrollada por Cassirer hunde sus raíces en la propuesta Uexküll (1926; ESPARZA 2019). El biólogo establece que todo organismo interactúa con su entorno físico inmediato a partir del conjunto 
de las formaciones orgánicas que caracterizan al individuo. La capacidad del animal para adaptarse se debe a las habilidades de la especie para responder a las demandas del entorno. Por ello, el biólogo propone una investigación, como punto de partida que detalla cómo y cualés procesos fisiológicos hacen posible que el organismo responda a las demandas del entorno:

The task of biology consists in expanding in two directions: (1) by considering the part played by our body, and especially by our senseorgans and central nervous system, and (2) by studying the relations of other subjects (animals) to objects" (UEXKÜLL, 1926, p. xv).

Para el estonio, los órganos sensitivos del ser vivo cumplen una función de respuesta frente a los estímulos del medio; así, el cuerpo del individuo conforma sus reacciones ambientales al organismo y viceversa. De esta conformidad Uexküll (1926, p. xi) planteará que toda especie se constituye de acuerdo a un "plan" de desarrollo orgánico. La base de esta conformación biológica, explica el estonio, se aprecia mejor a través de los recursos neurológicos a través de los cuales el individuo diferencia entre los estímulos propios y los del entorno. La capacidad de distinción entre percepciones que refieren a la propia estructura de aquellas que orientan al contexto, se identifican como el factor funcional que ha llevado a las especies desarrollar sistemas de reacción para vincular las necesidades corporales con el entorno físico.

Uexküll (1926), por ello, estudia el "Espacio" (UEXKÜLL, 1926, p. 1-51), el "Tiempo" (UEXKÜLL, 1926, p. 52-69) y los resultados los enmarca en una investigación sobre las "Cualidades del contenido" (UEXKÜLL, 1926, p. 70-86) desde el punto de vista biológico, haciendo explícito que cada individuo opera de acuerdo a un plan particular que no responde a leyes universales - como en el caso de la física -, sino que sigue leyes biológicas que explican las diferencias entre los organismos; por medio de este proceso se revela el mundo vital o "círculo funcional" de la especie. La consideración de la forma orgánica del individuo se traduce en el único medio posible de relación con el que cuenta el animal para adaptarse a su entorno. En parte ello explica por qué la interacción del ser vivo es irreducible a un contexto matemático, físico o químico, siendo necesarias las condiciones anatómicas de la especie para comprender su forma animal. La investigación fisiológica del individuo correspondería a un estudio de las características esenciales de la especie en lugar de una descripción de las características accidentales: "The anatomical structure of the sense-organs of animals brings together as a unity 
those indications that our attention also treats as a unified quality-circle" (UEXKÜLL, 1927, p. 86).

Sobre esta relación entre autores, Carlo Brentari (2015, p. 191-193) menciona que son tres los factores de incidencia de la biología vitalista uexkülliana en la epistemología cassireriana: (1) el estudio del medio ambiental en el que viven los animales no es reducible a un antropomorfismo desde donde se pretenda establecer condiciones generales de percepción aplicables a los seres vivos: cada animal percibe de acuerdo a su estructura corporal; (2) el interés metodológico del estonio por diferenciar entre el estudio de la estructura orgánica del ser vivo y su forma. Esta diferenciación no implica separación, sino que la distinción se encuentra correlacionada entre sí en contexto vital donde la especie se desarrolla; (3) la posibilidad de investigar el universo biológico a partir del individuo de la especie. La unidad de la especie no se refleja en la integración de todos los miembros, sino en la coordinación funcional del singular con el universal. Así, el estudio del individuo de la especie ofrece medios conceptuales plausibles derivar postulados generales

De este vitalismo uexkülliano, Cassirer retomará la advertencia de que toda especie opera dentro de un límite corporal (mundo interno), que tanto delimita como promueve el conjunto de acciones posibles del animal (mundo de la acción) dentro de un marco cultural de sentido (mundo circundante). Lo anterior permite al neokantiano delinear el conjunto de las acciones propias de la especie y de los individuos que a ella pertenecen; Cassirer sintetizará esta idea diciendo que en el mundo de las moscas encontraremos sólo cosas de mosca, en el mundo de los erizos de mar, se advierten sólo cosas de erizos de mar por lo que, en el mundo humano, existirá la cultura como producto natural (AEM, p. 41-45).

$\mathrm{Al}$ establecer que el ser humano, al igual que resto de las especies vivas, existe y se desarrolla en su propio "círculo funcional" (UEXKÜLL, 1916, p. 126-177; $A E M$, p. 41-44), el neokantiano sostendrá que en el mundo humano se conforma de operaciones propiamente "humanas". Frente a la necesidad de responder “¿Qué es lo humano?” (ZLK, p. 32-49; PSF, IV, p. 56-111), Cassirer se preguntará si la estructura natural del ser humano puede ser considerada como una de sus características esenciales. Los resultados filosóficos afirmaran que "el organismo no es un conglomerado de diversas partes, sino un sistema de funciones que se condicionan mutuamente. El tipo de este entrelazamiento revela clara y directamente el 'plan de construcción' de cualquier animal” (ZLK, p. 36). 
El cuerpo animal, entonces, es la expresión orgánica de la vida. La corporalidad humana no se entiende a través de las respuestas biológicas que se generan frente al medio que le circunda, sino que operará de acuerdo a una función nueva que le permite al ser humano, a un mismo tiempo, mantenerse atado al entorno material para liberarse de sus limitaciones orgánicas ${ }^{4}$. Por medio de la construcción de simbolizaciones formales (mito, lenguaje, ciencia), el hombre creará vínculos que le garantizarán actuar en relación a su organismo, pero a través de recursos que no son dados de un modo material (ZLK, p. 36-40).

Conviene aquí retomar la patología de la conciencia simbólica ofrecido en PSF, III (p. 241-327/234-322) con el fin de comprender la capacidad del organismo humano para construir símbolos. Para el autor, tanto la "afasia" y la "apraxia" (como enfermedad del habla y la conducta) se traducen en una pérdida de capacidad espiritual; para el enfermo, toda la visión teleológica de la acción se vuelve incomprensible, por lo que le resulta imposible la articulación temporal entre lo meramente inmediato y lo posible o final. A través del análisis de diversos casos en los que se esperan conductas de una persona "sana" se concluye que quien padece de este daño está ligado al mundo físico siendo incapaz operar su representación ${ }^{5}$. El carácter representativo del conocimiento se ha confinado a su presencia: "Lo que tenemos ante nosotros no es la pérdida de una facultad, sino el camino y la transformación de un proceso psíquico-espiritual altamente complejo (Was wir hier vor uns haben, ist nicht der Verlust eines Vermögens, sondern die Wandlung und Umbildung eines höchst komplexen psychisch-geistigen Prozesses)" (PSF, III, p. 324/318).

\footnotetext{
${ }^{4}$ Cassirer insistirá en este aspecto procurando subrayar que la conciencia animal del ser humano no es sólo capacidad de recepción de estímulos (como el resto de los animales), sino conciencia de los estímulos recibidos por parte de un medio-no-humano. La diferenciación del "yo" frente a lo "otro" (tú/ello), permite al individuo auto-conciencia de su estructura corporal como conciencia de la vida individual, la cual ni es una extensión del mundo circundante ni tampoco su oposición, sino una relación íntima en la que se expresa la experiencia de la vida: "The consciousness is a consciousness of objects insofar as it is self-consciousness- and it is selfconsciousness only in and by virtue of the fact that is a consciousness of objects... these determinations can be grasped only as existing "in addition" to each other; we have here $[\ldots]$ a correlation aspects" (PSF, IV, p. 66).

${ }^{5}$ Uno de los ejemplos que el autor presenta es el siguiente: "Der Gesunde führt die Bewegung des Einschlagens des Nagels ebensowohl gegen eine bloss „vorgestelle“ wie gegen di wirkliche Wand hin aus, weil er in reier Tätigkeit die Elemente des Sinnlich-Gegebenen zu variien vermag, veil er „in Gedanken" ein hier und jetzt Vorhandenes mit einemanderen Nichtvorhandenen vertauschen un das letztere an Stelle des ersteren setze kann” (PSF, III, p. 314). La diferencia entre un caso y otro, según el autor, es la posibilidad de operar en ciertos espacios imaginarios aplicando las mismas acciones que se desarrollarían en entornos físicos que cumplen con todas las condiciones de normalidad, pero en entornos virtuales.
} 
El autor, eventualmente, aclara que su investigación no busca rasgos comunes que soporten la personalidad, sino un sentido espiritual alcanzando a través del cuerpo humano. El neokantiano afirma que la patología de la conciencia simbólica se expresa en el sometimiento del sujeto a la cercanía de la vida, a la reducción de las funciones orgánicas a su pura operación en lugar de a la vinculación de dichas tareas con otros medios de relación espacial y temporal que posibiliten la vida, no como un suceso biológico reducido, sino como un suceso orgánico de interacción funcional. Según el neokantiano, el acto de advertir que se está vivo constituye una ruptura con el simple acto de vivir, pues esta se agota en la operación orgánica de la existencia. Cassirer concluye su tratado sobre la patología de la conciencia simbólica del siguiente modo:

\begin{abstract}
Elvalor y el significado de ese proceso de espiritualización, de "simbolización" del mundo se tornan justamente aprehensibles para nosotros en los casos en que ese proceso ya no se efectúa libre de obstáculos, sino que tiene que luchar y afirmarse en contra de impedimentos. En este sentido nos ofrecen la patología del lenguaje y de la conducta un patrón para medir la distancia que existe entre el mundo orgánico y el mundo de la cultura humana, entre el ámbito de la vida y el de 'espíritu objetivo' (In diesem Sinne geen uns die Sprachpathologie un die Pathologie des Handelns einen Maßtab in die Hand, mit dem wir die Breite des Abstandes messen können, der zwischen der organischen Welt un der Welt der menschlichen Kultur, zwischen dem Gebiet des Lebens un dem des 'objektiven Geistes' besteht)" (PSF, III, p. 327/322).
\end{abstract}

Este fragmento, es importante porque la patología del lenguaje constituye un recurso que permite medir (Maßtab) la identidad entre el organismo y su relación necesaria con el mundo del espíritu (Geist). Por ello, la formación simbólica del mundo no es una característica del organismo vivo, sino el resultado de una iteración funcional que se soporta en una forma corporal específica como la del ser humano y que, en un desarrollo progresivo y dialéctico, se logra la representación y significatividad del mundo circundante. Lo que posibilita el conocimiento del ser humano son los símbolos que surgen del entrelazamiento cuerpo-alma; a través de las vinculaciones entre el organismo, sus capacidades lógicas y psicológicas con el entorno, se alcanza la conexión entre el ser individual con una ley general que permite valorar la acción como: (i) una acción natural - propia del organismo que responde -, (ii) dentro de un entorno cultural - propia del organismo humano. 
Esta interrelación entre la "naturaleza orgánica" del individuo y el "entorno geográfico" en el que se desarrolla una especie, es reflexionada por Cassirer en PSF, IV (p. 3-33). Para él la forma de la vida (Leben) se encuentra íntimamente ligada con la forma espiritual (Geist) de tal modo que una reflexión sobre las condiciones naturales vincula directamente con la naturaleza espiritual que anima y posibilita la existencia del ser vivo: "Life as such appears to mean nothing other tan a pure kind of internal being; in fact its basic carácter appears to be defined by this very Being-in-itself and Reaminingin-itself" ( $P S F, \mathrm{IV}$, p. 9). Todos los esfuerzos por separar y comprender una realidad como ajena o independiente de la otra constituyen un contrasentido y esfuerzos infructuosos. Sólo en la comprensión de que una investigación biológica o filosófica se interesa por focalizar un aspecto de la realidad sobre otro - sin por ello entenderlos como realidades separadas - se podrá visualizar la estructura compleja que constituye la vitalidad y espiritualidad de la especie: "This distinction [between organic life and cultural spirituality] does not refer therefore to two metaphysical potentialities...instead, we only meet up with two accents that we fin in the process of becoming" (PSF, IV, p. 15).

$\mathrm{Si}$ bien Cassirer distingue el mundo natural y el mundo cultural ( $Z L K$, p. 81-120), el filósofo sostiene un proceso de construcción simbólico a través del cual se unen ambos mundos; frente a la necesidad de relacionar el mundo de la cultura a partir del mundo material, la forma corporal opera simbólicamente conjuntando el mundo espiritual de la cultura en el marco de su forma orgánica; el ser humano vive en la cultura. En este entrelazamiento no existe disolución de fuerzas, sino unidad relacional. Para María García (1998) la filosofía de Ernst Cassirer presenta a la cultura como universo simbólico que expresa la unidad funcional entre la forma orgánica (cuerpo) y la forma espiritual (alma) del ser humano (AEM, p. 278-286).

Con esto se ha mostrado que existe una relación de unidad entre cuerpo, alma y cultura. La tarea que resta es explicar cómo es que se expresa esta unidad en cada una de las fases del proceso fenomenológico del conocimiento.

\section{LA ENCARNACIÓN ESPACIAL Y TEMPORAL DEL MITO}

En la concepción mítica, el cuerpo encuentra un primer referente conceptual en la ubicación espacial, aunque también una experiencia de encuentro personal. El "yo" (que se concibe como existe en un entorno geográfico específico) advierte lo espacial como parte de una expresión 
anatómico-mágica. El individuo que se reconoce como aquél ser que está situado "aquî", refiere siempre a una posición del propio cuerpo dentro de un referente totémico. Todo el sistema de relaciones sociales en el que un individuo opera con un doble sentido: emotivo ( $A E M$, p. 97-142) y cósmico: "el mito parte de una concordancia espacio-física (räumlich-physichen) entre el mundo (Welt) y lo humano (Mensch) para inferir de esta concordancia la unidad de origen (Ursprungs)" (PSF, II, p. 125/107).

Si el individuo se sabe situado es porque existen recursos tribales o grupales designados como unidades totémicas espaciales que deben ser resguardadas (lo santo) o evitadas (lo profano) (PSF, II, p, 105-115). Es cierto que la existencia de entornos físicos por sí mismos no se constituyen como unidades culturales, sino que en todos los casos existe una función iterativa que establece que un lugar específico es un espacio "sagrado" o "profano" que se vincula con una forma general la cual se refiere a una símbolo totémico o divino (PSF, II, p. 228-243/213-229). Por ello, el referente material es siempre un signo que descansa en una concepción emotiva que promueve la aproximación o el rechazo. En el mundo mitológico "cada cosa y cada fenómeno es 'entendido' al incorporarse [a un] sistema totémico de clases y al dotársele de algún emblema totémico característico” (PSF, II, p. 119/101).

Entre el signo y el símbolo mitológico además de la función de significado construido culturalmente es necesaria la vinculación entre la cosa física y el contenido espiritual otorgado culturalmente. La existencia física de los objetos no depende de la carga emotiva con la cual experimentamos aquella subsistencia, sin embargo, es a través de esta emotividad que se advierte la forma del entorno físico. Ahora bien, la advertencia fenoménica de los espacios como sagrados y profanos depende de la cultura pedagógica que fomenta la iteración de las emociones que provienen del encuentro con diversas realidades del mundo material; al considerar a la comunidad como el marco de formación de sus integrantes es posible reconocer a la cultura como el recurso de relación entre las experiencias emotivas a través de las cuales se conforman los miembros del grupo social (PSF, II, p. 128-140/110-122).

La espacialidad mítica es el medio físico a través del cual se alcanza el reconocimiento de la propia estructura corporal; el entorno geográfico es el entorno donde lo corpóreo se hace presente en la medida en que se ubica, diferencia y reconoce como parte de un grupo totémico cultural. A través de esta referencia física se apertura un espacio mágico que se reconoce como un sentido espiritual que señala el carácter sacro o profano ( $P S F, \mathrm{II} ; A E M)$. 
A través de esta dualidad y su progresiva delimitación material, el individuo distingue entre aquello que está arriba, abajo, a la izquierda o a la derecha, como el producto de una oposición del propio cuerpo con el tótem. De este modo se construye la unidad funcional del espacio vital del individuo dentro de la tribu (PSF, II, p. 128-140/110-122). Con la disolución del espacio y su reunión en un sentimiento comunitario se ubica la experiencia orgánica personal (el sentimiento del propio organismo) y la experiencia del mundo físico (el sentimiento de otros organismos u objetos materiales) como un cosmos simbólico (García, 1998). Derivada de estas experiencias emotivas, el sujeto, a través de su cuerpo, advierte los espacios posibles de actuación, así como los entornos físicos a los que se debe negar.

Cassirer considera que, si bien la ubicación del sujeto a través de la experiencia corporal se refiere a un momento de singularidad, de la relación funcional de los instantes se revela la experiencia de lo temporal como unidad coherente de estabilidad (PSF, II, p. 140-156/123-140). En el marco de esta experiencia vital el espacio y el tiempo operan como actos psicológicos de un agente que actúa en relación a un todo comunitario.

En las descripciones de los distintos pueblos desarrolladas por el autor (PSF, II, p. 157-180/140-166) se aprecia que las acciones tribales se ordenan en relación a determinados sentimientos de fases (día/noche, izquierda/ derecha, adelante/atrás). El tiempo de los pueblos primitivos se divide por los ritos que se practican; los cambios importantes de la vida son acompañados de ritos que simbolizan los periodos de crisis. En el conjunto de las prácticas rituales subyace la idea de un tiempo biológico o cósmico (PSF, II, p. 105194/98-180).

Los sucesos individuales no son singularidades absolutas sino revelaciones instantáneas de la deidad, la cual dicta sus designios y deben ser operados por todos los seres. Este aspecto se profundiza en el trabajo de Ethel Junco (2016) quien sostiene que toda actividad mítica mantiene como trasfondo la intención de encuentro entre un ser humano y una deidad. La autora remarca que el "encuentro" es posible porque el que busca esta proximidad se ofrece corporalmente como signo de una entrega plena del propio ser; el sacrificio, resalta ella, representa el interés por expresar que el humano es un individuo concreto en busca de una respuesta universal, la cual se revela en el cumplimiento de un mandato divino. 
El tiempo, entonces, se hace visible por medio de la repetición de un rito en cuyo continuo acto de reiteración se advierte al cuerpo como recurso de cumplimiento de una tarea fijada como destino o naturaleza humana. La temporalidad es traducible a un momento de comprensión en donde el sujeto se identifica como ser orgánico que debe actuar para cumplir las acciones propias de su especie. Así, los círculos funcionales propuestos por Uexküll (1926) y conceptuados como el mundo (1) interno, de la (2) acción y (3) el circundante, se traducen correlativamente como el mundo del (a) autoconocimiento (b) del cuerpo y (c) de la comprensión del entorno natural, propio del ser humano.

\section{LA CODIFICACIÓN LINGÜÍSTICA DEL CUERPO: MIMESIS, ANALOGÍA Y SIMBOLIZACIÓN}

Cassirer no diferencia sustantivamente entre mito y lenguaje (Cassirer, 1946); ambas simbolizaciones se conforman paulatinamente en un origen común. Sin embargo, él acepta que el momento expresivo, frente a la incapacidad para representar los símbolos emotivos universales, debe apoyarse de una simbolización lingüística con el fin de estabilizar los el continuo de experiencias universales (PSF, I, p. 17-18/6-7). Mientras que los símbolos mitológicos se encuentran íntimamente circunscritos a las capacidades perceptivas del sujeto, los símbolos lingüísticos se sostienen de signos codificables culturalmente (AEM, p. 87-96).

Según Cassirer, el progreso del lenguaje comienza como actividad que hace presentes a los objetos; aquello "esto" señalado con la mano o lo palpado con el cuerpo, no sólo es la "cosa", sino el señalamiento de la cosa. A diferencia del mito en el que lo seńalado es la cosa, en el lenguaje la designación fonética de un objeto como "algo" constituye una primera toma de distancia intelectual de lo ofrecido. A esta fase, el autor le llamará "mimética" en la medida en que la palabra presenta al objeto (PSF, I, p. 148-153/137-142).

El continuo presentar los objetos corporalmente evoluciona al señalamiento de los objetos fonéticamente. La voz-signo constituye un momento de progresión espiritual en la medida en que la cosa ya no se conoce por su presencia, sino por su evocación; aquí la cosa es lo que de ella se expresa. A esta fase el autor le llamará fase "analógica" (PSF, I, p. 153-156/142-145).

En fase de la "simbolización", el sonido puede seguir presente, pero los conceptos operan como la representación de lo fonético y de la cosa material. 
El código conceptual es la representación significativa de la cosa, ya no se requiere ni la presentación, ni su representación, el objeto se hace presente por su pura referencia conceptual (PSF, I, p. 156-158/145-146).

Es importante anotar que esta caracterización del lenguaje de la "mímesis" hacia la "analogía" y su culmen en la "simbolización", no se desarrolla al margen de las mismas categorías que revisten al mito, sino que también operan progresivamente los recursos espaciales y temporales en el desarrollo y cohesión de los códigos lingüísticos. Las etapas primarias de esta forma simbólica se caracterizan por un apoyo marcado del cuerpo por presentar los objetos materiales.

[L]a intuición del propio yo, del alma de la persona (die Anschauung des Selbst, der Seele, der Person dort) empieza a brillar en el lenguaje, sigue todavía íntimamente ligada al cuerpo (Körpen), así como también en la intuición mítica, el alma y el yo del hombre (die Seele und das Selbst des Menschen) son pensados en un principio como una mera réplica, como 'dobles' del cuerpo (als 'Doppelgänger' des Leibes gedacht wird) (PSF, I, p. 227/215-216).

Esta advertencia de relación en cuanto copia que se mantiene pegada, constituye el fundamento a través del cual la eventual representación es posible. $\mathrm{Si}$ las expresiones lingüísticas re-presentan, es porque existe un substrato presentable, el cual se caracteriza por su forma orgánica.

Esto anterior se ve con claridad cuando el neokantiano estudia las expresiones manuales, corporales, así como el uso de gestos, de señas, como el medio para la configuración de un objeto a partir de la mímica, aun cuando, eventualmente, la configuración de los recursos analógicos que permitan representar, ya no el objeto físico sino las propias gesticulaciones como signos de la cosa. Aunque en el lenguaje el intermedio no es una acción corporal directa, opera como una representación corporizada, fonética o escrita, que permite la representación (Darstellung) inmaterial del objeto (PSF, I, p. 224259/212-248). El paulatino progreso analógico, a decir de Cassirer, constituye un esfuerzo por superar la presencia del gesto físico para constituirse en un signo puro o escritura, la cual, en lugar de procurar presentar la cosa material, se ofrece un sentido que permita prescindir del objeto para retornar a él ( $P S F$, I, p. 260-280/249-269).

Para el neokantiano la simbolización formal del lenguaje se completa cuando se otorga sentido cultural a los signos empleados culturalmente. El proceso se deriva de una repetición progresiva que favorece la incorporación de 
sentidos y significados a los sonidos y signos. Aquí las palabras no representan a través del sonido físico, sino por medio de las relaciones lógicas que articulan la "palabra”, “intención”, "emoción” y las normas "culturales”, lo que en su conjunto constituye el "sentido" general del lenguaje expresado.

\section{LAS REPRESENTACIONES CIENTÍFICAS: IDENTIDAD ENTRE CUERPO Y CULTURA}

Las formas de relación existentes entre el cuerpo y la cultura que se desarrollan en la ciencia, constituyen uno de los intereses más recurrentes a lo largo de la filosofía de Cassirer (SuF, p. 271-307/359-408; PSF, III, p. 115129/104-118; $Z L K$, p. 121-141), sin embargo, mostraré la plausibilidad de que el "cuerpo", en tanto que forma orgánica del ser humano, es necesario para el desarrollo de los símbolos científicos.

Según el neokantiano la función es una operación lógica de identidad entre "miembros de una serie" y "forma de la serie" para acentuar que, aún en el concepto científico, permanece, idealmente, el cuerpo humano. Esta relación no se aprecia de un modo explícito en la obra de este autor, por lo que se tiene que interpretar un camino para mostrar esta relación.

Si se considera que $S u F$ presenta los fundamentos lógicos del conocimiento de toda la obra de Cassirer, parecería como si las simbolizaciones formales se ajustaran a un "formalismo" epistémico en el cual no hay cabida para el cuerpo humano en cuanto realidad material concreta; es decir, la estructura corporal, para la ciencia, parece estar supeditada a la forma de la ciencia, careciendo de interés la forma del organismo. Sin embargo, es importante recordar que el planteamiento que el autor hace al inicio de PSF, III:

Las investigaciones de mi obra Concepto de Sustancia y concepto de función parten de que la constitución básica del conocimiento y su ley (Gesetz) constitutiva puede descubrirse con mayor claridad y agudeza ahí donde el conocimiento ha alcanzado su más alto nivel de 'necesidad' y 'universalidad' ('Notwendigkeit' und 'Allgemeinheit') [...] La filosofia de las formas simbólicas ha ido más allá de este planteamiento inicial del problema tanto en cuanto al contenido como en cuanto al método. Ella ha ampliado el propio concepto fundamental de teoría al tratar de probar que no sólo son auténticos factores y motivos formales los que imperan en la configuración de la imagen científica del mundo (Gestaltung des wissenschaftlichen), sino también los que ya existen en la configuración de la 'imagen natural del mundo' la imagen de la percepción y la intuición (der Gestaltung des natürlichen Weltbildes, des Weltbildes der Wahrnehmung und Anschauung) (PSF, III, p. 7/VII). 
Con esta afirmación, el autor considera que el mundo natural se constituye como "imagen" no en razón a su existencia, sino en relación con el sujeto cognoscente que la percibe. Si la "imagen" que se logra en la ciencia, inicialmente, se aclaraba en la Física y en la Matemática esto se debía a un progresivo desarrollo histórico que el autor describiría en algunas de sus obras, pero que no determinaban la forma de la ciencia como tal. Todo el camino fenomenológico por el que debía avanzar la ciencia, desde sus estadios míticos y lingüísticos y hasta los científicos, reflejan los medios de representación de los estadios previos. De este modo, la "imagen teorética", en lugar de oponerse a la "imagen natural", encuentra armonía en la tensión progresiva que entre ambas existe. De ese modo, la ciencia, en cuanto símbolo, mantiene de modo relacional la realidad material del cuerpo que si bien se propone mantener en cuanto referente cósico de validación, no se empeña en mantenerlo en su realidad orgánica, sino que procurará "representarlo" en su forma orgánica.

En este punto - y siguiendo el planteamiento de Andoni Ibarra y Thomas Mormann (2019, p. 5. Énfasis añadido) - es importante recordar que las representaciones científicas son "sistemas mixtos de representaciones que combinan representaciones matemáticas con representaciones materiales de distintos tipos", ya que su objetivo es la subrogación de las inferencias y resultados en el dominio representante hacia el dominio representado. De ese modo - del continuo transferir resultados de un dominio a otro - es comprensible que la estructura del cuerpo no aparezca como realidad material (como en el caso del mito) o sígnica (como en el caso del lenguaje), sino como forma orgánica. El cuerpo humano, en la ciencia, no está presente de modo material sino a través de su simbolización permitiendo su estudio y la subrogación de los resultados científicos a través de la armonía preestablecida entre la forma y la realidad.

El propio Cassirer, en su esfuerzo por explicar la función de la ciencia dentro del sistema de las simbolizaciones formales, plantea que el desarrollo fenomenológico de este símbolo comienza con el acto de percibir o de preñar simbólicamente los sucesos cotidianos, para luego avanzar hacia configuraciones conceptuales que no se caracterizan por negar el entorno material, sino por las iteraciones formales $\mathrm{u}$ homológicas con las cuales se construyen las imágenes significativas propias de la ciencia (IBARRA Y MORMANN, 2019, p. 1320).

Ya el propio Cassirer (PSF, III, p. 12/1) muestra que la percepción es una capacidad del sujeto y es siempre intencional, por lo que la percepción 
siempre entraña un "índice de refracción" peculiar, por lo que "no hay 'materia en sî ni 'forma en sî sino sólo vivencias globales que pueden compararse desde el punto de vista de materia y forma y [...] determinarse y articularse" (CASSIRER PSF, III, p. 235/227). Por lo que, a decir del neokantiano, "no sólo proyectamos emotivamente nuestros propios estados interiores de modo arbitrariamente subjetivo en la forma espacial, sino ella misma se nos da como totalidad animada, como manifestación independiente" (CASSIRER PSF, III, p. 236/228).

En este contexto, el cuerpo no es lo que se opone al mundo espiritual, sino el estatuto fundamental para el desarrollo de la cultura, la cual, opera en función de la biología humana. Esto se puede apreciar si recordamos los siguientes puntos: a) la estructura corporal de cualquier especie constituye su delimitación material, b) todas estas definiciones se explican a partir de las fronteras corporales y c) la existencia del ser vivo se confina por sus límites anatómicos. Aplicados estos puntos al caso del ser humano, se advierte que, (i) la función orgánica se encuentra definida (y limitada) por el cuerpo, (ii) a través del cual el individuo opera como un ser humano vivo. Así, (iii) la obra cultural se constituye como expresión espiritual sólo en la medida en que permanece unida al cuerpo.

Al respecto, Cassirer considera que esta unidad de relación entre lo corporal y lo cultural en la ciencia, mantienen unidad de función sin por ello ser perceptible o evidente:

Aunque haya desaparecido bajo su forma concreta y específica, ha ejercido antes de desaparecer efectos que dejan una huella que influyen de algún modo en la trayectoria de la cultura y que tal vez contribuyen decisivamente a ella en alguno de sus puntos. [...] La razón de ello está en que la humanidad, con su lenguaje, su arte, con todas sus formas de cultura, se crea en cierto modo un nuevo cuerpo, que pertenece en común a cuantos la forman ( $Z L K$, p. 175).

De este modo, las relaciones entre corporalidad y ciencia no se reflejarán por la materialidad del organismo humano, sino por su relación funcional y la vinculación que existe entre la naturaleza y el espíritu, por la tensión armónica que caracteriza la distancia de los opuestos que se encuentran unidos culturalmente, pero que sólo se comprende por medio de una filosofía que se proponga delimitar los procesos corporales de simbolización y sus formas culturales de manifestarse (CASSIRER, 1949). 


\section{EL CUERPO COMO SÍMBOLO Y SU LUGAR DENTRO DE LA INVESTIGACIÓN ACTUAL}

Antes de finalizar con estas reflexiones es importante contextuar el lugar que estas reflexiones ocupan dentro del estado de la cuestión actual que se interesa por reflexionar sobre el problema del cuerpo. De forma sintética, podemos identificar cuatro grandes grupos de problemas: (1) indagaciones sobre la naturaleza unitaria de lo corporal; (2) investigaciones sobre las condiciones pedagógicas que indagan sobre el cuerpo; (3) reflexiones sobre el lugar que ocupa el cuerpo humano dentro de los avances tecnológicos; (4) cuestiones éticas sobre la naturaleza del cuerpo.

El primer grupo se cuestiona si el cuerpo puede considerarse como una parte constitutiva de la naturaleza humana o si, por el contrario, se trata de una naturaleza distinta que coexiste ya sea en armonía o tensión permanente. Este problema platónico propuesto en el Fedón ha sido retomado por Descartes en sus Meditaciones Metafísicas y actualmente por una gran variedad de autores, pero cuyo motivo de reflexión central es la reflexión sobre la posible unidad alma-cuerpo. Al respecto, podemos reconocer el aporte específico de Cassirer a esta discusión: el cuerpo es una unidad simbólica que asume la unidad alma-cuerpo como una realidad que coexiste en armonía de contrarios (BENEDICTO, 2012; GONZÁLEZ, 2015; CHARLTON 2019; PSF, III).

El segundo conjunto de trabajos asume la unidad integral del cuerpo como una tarea pendiente. En lugar de problematizar la posible relación entre las facultades del alma y sus correlatos orgánicos, este grupo de trabajos considera que la historia del pensamiento está en deuda con los medios que hacen posible su potencialización y, sobre todo su formación humana. El centro de estas indagaciones asume que el cuerpo es una condición pedagógica indispensable para la formación del ser humano. Si bien Cassirer no indagó directamente sobre las condiciones pedagógicas (a diferencia de su maestro Natorp (1975) quien desarrollo una teoría al respecto), podemos entender que en aquellos casos en los que existe una atrofia que implica una disolución de la unidad simbólica entre las facultades y la estructura biológica del cuerpo, esto ha de traducirse como un impedimento en el pleno desarrollo del individuo, así como de sus capacidades adaptativas (MILLÁN, 2012; AYALA, NOREÑA, SANABRIA, 2015; ESPARZA 2019; PSF, III).

El tercer grupo reflexiona sobre las relaciones entre el cuerpo y el lugar que ocupa éste como realidad física dentro de un mundo que indaga, evoluciona de la mano de los avances tecnológicos. La cuestión central es reflexionar si el 
ser humano, en cuanto criatura orgánica inteligente y capaz de adaptarse a las demandas de su entorno, puede asumir los avances creados por él mismo como un constitutivo natural de su forma orgánica. De este modo, las innovaciones tecnológicas parecen operar como una realidad propia del individuo. En este punto, para Cassirer - aunque ajeno en su tiempo a estas reflexiones - aporta a la discusión al situar al ser humano como un "animal simbólico" que debe operar y desarrollarse a través de su realidad orgánica, pero reconociendo sus capacidades internas como un agente capaz de crear e innovar. Sin embargo, la propuesta del autor no se encuentra en asumir las novedades tecnológicas como un elemento inherente al ser humano, sino como una aportación a la cultura. En ese sentido, se debe entender las diferencias entre lo cultural y lo humano que, aunque se unen simbólicamente se diferencian en cuanto realidades opuestas que interaccionan armónicamente (SÁNCHEZ, 2010; PARRA, BARRIOS, 2015; ESPARZA 2019; AEM).

El cuarto grupo reflexiona sobre las condiciones de posibilidad de una práctica ética por parte del individuo. Considerando que gran parte de las acciones humanas descansan en su capacidad para actuar de un modo consciente, algunas de las investigaciones se han cuestionado si existen condiciones neurológicas para establecer una voluntad inmaterial o distinta a la realidad cerebral. En ese sentido, las reflexiones orientan sus cuestionamientos a las posibilidades de asumir una moralidad de las acciones en lugar de postular una neurobiología de las decisiones. En contraparte, para Cassirer, si bien el cuerpo es fundamental al momento de operar conscientemente, él mismo considera que únicamente la unidad simbólica del ser humano, su plena naturaleza como animal simbolizante permitirá entender tanto la libertad de la que está dotado, como su capacidad de agencia para construir una cultura humana (CHURCHLAND, 2012, FIGUEROA, 2013; AEM).

En general, se puede afirmar que el peligro, para este autor, no radica en la fragmentación del ser humano para estudiarlo como un ser orgánico, animado o cultural, sino en suponer que dicha focalización en uno u otro aspecto de la naturaleza humana pueden asumirse y reflejarse como realidades en contraposición. La insistencia central del filósofo de Breslavia fue llamar a una unidad simbólica del cuerpo y el alma porque de otro modo no sería posible entender la estructura actual de nuestra cultura. Por ello, aunque encontramos algunos pasajes en los que se sistemátiza el problema del cuerpo, el tema aparecerá reiteradamente en toda la obra del autor porque desde los diversos puntos de vista abordados, se expone la corporalidad como una 
realidad básica desde la cual había de partir para comprender problemas complejos posteriores (como el mito, el lenguaje y la ciencia). Sólo desde la reflexión de la unidad cuerpo-alma-cultura es posible entender al ser humano como un ser cultural.

\section{ConClusiones}

En el presente trabajo se profundizó en los conceptos de "cuerpo", "alma" y "cultura" en la filosofía de Ernst Cassirer para exponer cómo era posible la unidad triple dentro de la Fenomenología del conocimiento. Se resaltaron cuatro resultados sobre este punto: primero, la designación de las relaciones entre el cuerpo y el alma como un fenómeno originario, propio de la función expresiva. Segundo, la relación de unidad cuerpo-alma, se extiende a lo largo del proceso fenomenológico descrito por el neokantiano (representación y significación). Tercero, se reconoce que el cuerpo es una forma necesaria para la construcción del conocimiento, por lo que la unidad de lo físico y lo espiritual opera como condición indispensable para el desarrollo de una Fenomenología del conocimiento. Sin embargo, no existía un estudio en el que se expresara la unidad cuerpo-alma-cultura en este progreso epistemológico. Cuarto, las simbolizaciones formales adquieren sus formas simbólicas particulares debido al círculo funcional del organismo que las construye. Por ello, el tránsito de lo singular corpóreo a lo universal del espíritu adquiere la única "forma" armónica coherente y derivable de la estructura del organismo humano: la forma de la cultura.

Como objetivos particulares fueron expuestos los vínculos corpóreos con las fases expresiva, representativa y significativa. Los resultados muestran que las condiciones físicas del ser humano -su forma corpórea- cumple las siguientes tareas: en el mito, el cuerpo es la condición de desarrollo de una conciencia espacial y temporal; en el lenguaje sustenta la progresión representativa (mímesis, analogía y simbolización); y, en la ciencia, constituye el fundamento material para el desarrollo de los sistemas de relaciones.

Se hizo notar también que si bien las reflexiones de Cassirer se realizaron en la primera mitad del siglo pasado, sus aportaciones ya visualizaban una tendencia en las reflexiones que la temática del cuerpo asumiría a través del tiempo. La tesis central del autor sobre la unidad simbólica entre el cuerpoalma-cultura sirven como base para indagar y dialogar con las aportaciones contemporáneas acerca del papel que ocupa nuestra estructura orgánica dentro 
del conjunto de los problemas a los que nos enfrentamos cotidianamente. Si bien es importante continuar indagando sobre la naturaleza y estructura de la relación de nuestra realidad como seres humanos, podemos considerar la filosofía del cuerpo propuesta por el neokantiano como una propuesta valida que dista mucho de verse agotada en las reflexiones actuales.

ESPARZA, G. Body and symbol in Ersnt Cassirer: the organic function in the conformation of cultural wolrd. Trans/form/ação, Marília, v. 43, p. 205-230, 2020. Edição Especial.

\begin{abstract}
In this paper is studied the function of human body and its relation with the culture, in Ernst Cassirer's "Philosophy of Symbolic Forms". According to the neokantian, the relation soulbody conforms the basic relation to develop the Phenomenology of knowledge, nevertheless through the dialectic process is necessary to deny the material form to achieve the spiritual form of the culture, so the organic form, apparently, needs to be annulled in this dialectic progression. The aim is to analyze, and describe, how the form of the body appear in expressive mythical stage, but also in representative stage of language and significative stage of science, allowing the unity in the entire process of the construction of knowledge and the form of culture
\end{abstract}

Keywords: Body. Symbol. Knowledge. Organic function. Cultural function. Uexküll. Cassirer.

\title{
REFERENCIAS
}

AYALA, Marta; NOREÑA, Néstor; SANABRIA, Manuel. El cuerpo: un saber pedagógico pendiente. Tesis psicológica. Vol. 10, No. 2, p. 174-188, 2015.

BENEDICTO, Rubén. Martha Nussmbaum: Emociones, mente y cuerpo. Themata. Revista de Filosofía. Vol. 46, p. 591-598, 2012.

BRENTARI, Carlo. Jakob von Uexküll. The discovery of the Umwelt between Biosemiotics and the Theoretical Biology. New York: Springer, 2015.

CASSIRER, Ernst. An essay on man: an introduction to a philosophy of culture. New York: Doubleday Anchor, 1945.

CASSIRER, Ernst. Language and Myth. New York: Dover Publications, 1946.

CASSIRER, Ernst. Substance and Function. New York: Dover Publication, 1953 [Substanzbegriff und Funktionsbegriff. Untersuchungen über die Grundfragen der Erkenntniskritik. Berlin: Bruno Cassirer, 1910]. 
CASSIRER, Ernst. "Spirit" and "Life” in contemporary philosophy. En E. Schilpp, The Philosophy of Ernst Cassirer, Vol. VI. Evanston, Illinois: The Library of living philosophers, Inc., p. 857-880, 1949.

CASSIRER, Ernst. The philosophy of Symbolic Forms: the metaphysics of Symbolic Forms. New Haven \& London: Yale University, 1996.

CASSIRER, Ernst. Filosofía de las formas simbólicas: El lenguaje. México: Fondo de Cultura Económica, 1998a. [Philosophie der Symbolischen Formen: Die Sprache. Hamburg: Meiner, 2010].

CASSIRER, Ernst. Filosofía de las formas simbólicas: El pensamiento mítico. México: Fondo de Cultura Económica, 1998b [Philosophie der Symbolichen Formen: Das mythische Denken. Hamburg: Meiner, 2010].

CASSIRER, Ernst. Filosofía de las formas simbólicas. Fenomenología del conocimiento. México: Fondo de Cultura Económica, 1998c [Philosphie der Symbolischen Formen: Phänomenologie der Erkenntnis. Hamburg: Meiner, 2010].

CHARLTON, William. Is the mind-body relationship mysterious? Philosophy. Vol. 94, No. 4 (1), p. 673-685, 2019. https://doi.org/10.1017/S0031819119000305

CHURCHLAND, Patricia. El cerebro moral. Lo que la neurociencia nos cuenta sobre la moralidad. Barcelona: Paidos, 2012.

COHEN, Hermann. The syntetic principles, from a Kant's Theory of Experience (1885). En: Sebastian Luft, The Neo-kantian reader. New York: Routledge, 2015.

ESPARZA, Gustavo. Ernst Cassirer: una fundamentación biológica de la definición del ser humano como "animal simbólico". Open insight. Vol. 10, No. 18, p. 124-144, 2019. https://doi.org/10.23924/oi.v10n18a2019.pp125-144.289

FIGUEROA, Gustavo. Las ambiciones de la neuroética: fundar científicamente la moral. Acta Bioethica. Vol. 19, No. 2, 259-268. http://dx.doi.org/10.4067/S1726569X2013000200010

GARCÍA, María. La cultura como universo simbólico en la antropología de E. Cassirer. Pensamiento. N. 209, v. 98, p. 221-244, 1998.

GONZÁLEZ, Roberto. Apuntes para una filosofía de la mente a partir de Ernst Cassirer, En-claves del pensamiento. Vol. 9 (18), p. 57-77, 2015.

IBARRA, Andoni; MORMANN, Thomas. Una teoría combinatoria de las representaciones científicas. Revista Hispanoamericana de Filosofía. p. 3-46, 2000.

JUNCO, Ethel. Eurípides y la belleza del Bien. Zacatecas: Texere-Universidad Panamericana, 2016.

KÖHNKE, Klaus. Surgimiento y auge del neokantismo. La filosofía universitaria alemana entre el idealismo y el positivismo. México: Fondo de Cultura Económica, 2011. 
KROIS, John. Philosophical anthropology and the embodied cognition paradigm. On the convergence of two research programs. En. J. Krois, et al., Embodiment in Cognition and Culture. Amsterdam-Philadelphia: John Benjamins, p. 273-289, 2007. KROIS, John, ROSENGREN, Mats, STEIDELE, Angela, WESTERKAMP, Dirk. Embodiment in Cognition and Culture. Amsterdam-Philadelphia: John Benjamins, 2007.

MILLAN, Luz. Cuerpo y subjetividad: hacia una pedagogía desde lo corporal. Revista Multidisciplinaria del Consejo de Investigación de la Universidad de Oriente. Vol. 24, N. 2, p. 191-195, 2012.

NATORP, Paul. Propedéutica filosófica/Kant y la Escuela de Marburgo/Curso de Pedagogía Social. México: Porrúa, 1975.

PARRA, Omar; BARRIOS, Hernando. El transhumanismo: cuestión de piel. Escritos. Vol. 23, No. 50, p. 43-65, 2015.

RALÓN, Graciela. Una interpretación de la percepción: Cassirer-Meleau-Ponty. Tópicos. Vol. 22, p. 35-53, 2002.

ROSENGREN, Mats. Radical imagination and symbolic pregnance. A CastoriadisCassirer connection. En: J. Krois, et al., Embodiment in Cognition and Culture. Amsterdam-Philadelphia: John Benjamins, p. 261-272, 2007.

SÁNCHEZ, José. Cuerpo y tecnología. La virtualidad como espacio de acción contemporánea. Argumentos. Vol. 23, No. 62, 227-243, 2010.

UEXKÜLL, Jakob. Theoretical Biology. New York: Harcourt, Brace \& Company, 1926.

Recebido: 23/5/2018

Aceito: 23/10/2019 
ESPARZA, G. 